\title{
Diode Laser-Induced Fluorescence of Xenon Ion Velocity Distributions
}

\author{
Timothy B. Smith*, Bailo B. Ngom ${ }^{\dagger}$, Jesse A. Linnell ${ }^{\ddagger}$, and Alec D. Gallimore ${ }^{\S}$ \\ Plasmadynamics and Electric Propulsion Laboratory, University of Michigan, Ann Arbor, MI 48105
}

\begin{abstract}
We present preliminary results from optogalvanic and laser-induced fluorescence (LIF) measurements of the nuclear-spin and isotopic structure of the $5 d^{2} F_{7 / 2} \rightarrow 6 p^{2} D_{5 / 2}^{0}$ transition of singly-ionized xenon (Xe II) at $834.7 \mathrm{~nm}$. Ion velocity distributions deconvolved from $5 d^{4} D_{7 / 2} \rightarrow 6 p^{4} P_{5 / 2}^{0}$ optogalvanic spectra at $605.1 \mathrm{~nm}$ provided the Doppler broadening function for a computational model of the $834.7 \mathrm{~nm}$ absorption line. Ensemble averaging of optogalvanic spectra taken at $834.7 \mathrm{~nm}$ provided an experimental data set for estimation of the unknown isotopic shifts and lower-state $\left(5 d^{2} F_{7 / 2}\right)$ nuclear-spin constants via a conjugate-gradient optimization routine. Deconvolution of $834.7 \mathrm{~nm}$ LIF spectra with the newly-modeled $834.7 \mathrm{~nm}$ cold-plasma spectrum returned an unusually broad velocity distribution, rather than the expected kinematic cooling effect. Comparison of a deconvolved ion energy distribution taken in a $500 \mathrm{~V}$ ion beam with retarding potential analyzer data showed bulk ion energy agreement within $1 \%$, but only qualified similarity between the distributions. Grid misalignment during the LIF experiment may be responsible for the usually broad Doppler broadening. As a result, we are presently unable to reliably validate our deconvolution method at $834.7 \mathrm{~nm}$.
\end{abstract}

\section{Introduction}

Laser-induced fluorescence (LIF) velocimetry of thruster plumes and internal flows requires a tunable, narrow-linewidth laser. Dye lasers, though capable of reaching a wide range of wavelengths, are complex, expensive and difficult to operate. However, dye lasers provide the only light source capable of singlyionized xenon (Xe II) LIF driven by the $5 d^{4} D_{7 / 2} \rightarrow 6 p^{4} P_{5 / 2}^{0}$ absorption at $605.1 \mathrm{~nm}$. This absorption is the only Xe II transition that currently has a well-established hyperfine structure (HFS), permitting accurate computational modeling of the LIF spectrum. As a result, deconvolution of the LIF at $605.1 \mathrm{~nm}$ with a simulated cold-plasma spectrum ${ }^{1}$ is the only optical method currently available for high-spatial-resolution estimation of the Xe II velocity distribution.

External cavity diode lasers (ECDLs) are considerably less expensive and troublesome to operate than dye lasers, though more limited in wavelength range. Two neutral xenon (Xe I) absorptions $\left(6 s[3 / 2]_{2}^{0} \rightarrow 6 p[3 / 2]_{2}\right.$ at 823.2 and $6 s[3 / 2]_{1}^{0} \rightarrow 6 p[1 / 2]_{0}$ at $\left.828.0 \mathrm{~nm}\right)$ and one xenon ion (Xe II) absorption $\left(5 d^{2} F_{7 / 2} \rightarrow 6 p^{2} D_{5 / 2}^{0}\right.$ at $834.7 \mathrm{~nm}$ ) are readily probed by commercially-available diode lasers. ${ }^{2}$ The $834.7 \mathrm{~nm}$ transition has proven to be especially popular for bulk velocity measurements in internal ${ }^{3-7}$ and external ${ }^{8,9}$ Hall thruster flows. Unfortunately, the isotopic shifts and lower state $\left(5 d^{2} F_{7 / 2}\right)$ nuclear-spin structure constants are unknown for the $834.7 \mathrm{~nm}$ Xe II transition. Though the bulk velocity can be readily measured from the Doppler shift of the transition, the incomplete HFS model limits its usefulness in either deconvolving the ion velocity distribution or estimating its higher moments (such as the ion temperature) from the LIF spectrum.

In a series of Xe II optogalvanic spectrocopy (OGS) experiments carried out with a Hamamatsu L2783 xenon-neon galvatron, we have determined reasonable estimates of the missing lower-state $\left(5 d^{2} F_{7 / 2}\right.$ nuclearspin constants and isotopic shifts for the Xe II transition at $834.7 \mathrm{~nm}$. Deconvolution of 49 OGS spectra

\footnotetext{
*Research Fellow, Aerospace Engineering, Senior Member AIAA.

${ }^{\dagger}$ Graduate Student Research Assistant, Aerospace Engineering, Student Member AIAA.

${ }^{\ddagger}$ FXB Fellow, Aerospace Engineering, Student Member AIAA.

$\S$ Professor, Aerospace Engineering, Associate Fellow AIAA

Copyright (C) 2005 by the American Institute of Aeronautics and Astronautics, Inc. All rights reserved.
} 
recorded at $605.1 \mathrm{~nm}$ provided the Doppler broadening function for computational modeling of the $834.7 \mathrm{~nm}$ absorption line. A conjugate-gradient optimization routine then found values of the unknown HFS constants that minimized the mismatch between the model and the ensemble average of 61 OGS spectra at $834.7 \mathrm{~nm}$.

In a concurrent series of $834.7 \mathrm{~nm}$ LIF measurements in the Cathode Test Facility (CTF) at the University of Michigan Plasmadynamics and Electric Propulsion Laboratory (PEPL), we attempted to demonstrate kinematic cooling of Xe II by electrostatic acceleration through the grids of a Commonwealth Scientific 3 $\mathrm{cm}$ ion source. Though the hoped-for kinematic cooling failed to appear, we were able to deconvolve the resulting LIF spectra with the newly-modeled cold-plasma spectrum at $834.7 \mathrm{~nm}$. We compare the results of ion energy distributions estimated from LIF spectra and retarding potential analyzer (RPA) probing of the $3 \mathrm{~cm}$ ion source plume at a beam voltage of $500 \mathrm{~V}$.

\section{Theory}

In LIF, the wavelength of a narrow-linewidth tunable laser is swept through an absorption line of a plasma species, such as singly-ionized xenon (Xe II). Focusing optics direct this laser beam along a "beamwise" direction vector $\hat{\mathbf{k}} \equiv \mathbf{k} /|\mathbf{k}|$, where $\mathbf{k}$ is the beam's wave vector. Collection optics on another (usually perpendicular) axis sample the fluorescence emitted by absorbing particles in the interrogation volume. This interrogation volume, an ellipsoid defined by the intersecting beams, can be less than a millimeter on a side. Wavelength filtering and lock-in detection keep the LIF signal from being swamped by the bright light background typical of EP devices.

\section{A. Line broadening}

Consider a light source with vacuum wavelength $\lambda$ and frequency $\nu=c / \lambda$. An observer who is stationary with respect to the light source will see light at the same frequency $\nu$. An observer moving towards the light source will see a bluer (i.e., higher-frequency) light than the stationary viewer, while an observer moving away from the light source will see a redder (i.e., lower-frequency) light.

In LIF, this Doppler effect appears as a shift in the resonant frequency $\nu_{0}$ as the laser is scanned over a very short frequency range. The change in photon frequency $\Delta \nu=\nu-\nu_{0}$ for a particle with velocity $\mathbf{v}$ passing through a light beam of wave vector $\mathbf{k}$ is

$$
\triangle \nu=-\frac{\mathbf{k} \cdot \mathbf{v}}{2 \pi} .
$$

Given a beamwise velocity component $v_{k} \equiv \mathbf{v} \cdot \hat{\mathbf{k}}$, Eqn. 1 gives the beamwise velocity-to-frequency transformations

$$
\begin{aligned}
\nu & =\left(1-\frac{v_{k}}{c}\right) \nu_{0} \\
v_{k} & =\left(1-\frac{\nu}{\nu_{0}}\right) c .
\end{aligned}
$$

A swarm of particles with a normalized velocity distribution $f(\mathbf{v})$ will also "see" the frequency of incoming photons shifted by the relative velocity of the particle in the direction of the photon. The resulting Doppler lineshape will be shifted by the beamwise bulk velocity $u_{k}=\langle\mathbf{v} \cdot \hat{\mathbf{k}}\rangle$ and broadened by the thermal width of the beamwise distribution $f\left(v_{k}\right)$. The generalized Doppler lineshape $d(\nu)$, when properly normalized so that

$$
\int_{-\infty}^{\infty} d(\nu) d \nu=1
$$

is given by

$$
d(\nu)=\frac{c}{\nu_{0}} f\left(\left[1-\frac{\nu}{\nu_{0}}\right] c\right) .
$$

When $f\left(v_{k}\right)$ is a one-dimensional stationary Maxwellian of the form

$$
f_{m}\left(v_{k}\right)=\left(\frac{M}{2 \pi k T}\right)^{1 / 2} \exp \left(-\frac{M v_{k}^{2}}{2 k T}\right),
$$


Eqn. 5 takes the familiar form ${ }^{10}$

$$
d_{m}(\nu)=\frac{c}{\nu_{0}}\left(\frac{M}{2 \pi k T}\right)^{1 / 2} \exp \left(-\frac{M c^{2}}{2 k T}\left[\frac{\nu-\nu_{0}}{\nu_{0}}\right]^{2}\right) .
$$

In species with no hyperfine structure (HFS), the LIF spectrum $i(\nu)$ is the convolution of the Doppler broadening with the natural (or lifetime) broadening function

$$
l(\nu)=\frac{\triangle \nu_{n}}{2 \pi} \frac{1}{\left(\nu-\nu_{0}\right)^{2}+\left(\triangle \nu_{n} / 2\right)^{2}}
$$

where $\triangle \nu_{n}=A_{j} /(2 \pi)$ is the natural linewidth for state $j$. This Lorentzian function is usually much narrower for warm gases than the Doppler broadening. In this case, the natural broadening can be replaced by a Dirac delta function,

$$
i(\nu)=d(\nu) \otimes l(\nu) \approx d(\nu) \otimes \delta(\nu)=d(\nu),
$$

letting us directly transform the LIF spectrum into an excellent approximation of the beamwise velocity distribution. The similarity between LIF spectra and $f\left(v_{k}\right)$ is good enough that LIF measurements in barium $^{11-13}$ and $\operatorname{argon}^{14}$ plasmas have been reported as velocity distributions.

\section{B. Hyperfine structure}

In xenon (and other species with HFS), the LIF spectrum can be modeled as the convolution of the hyperfine splitting function $h(\nu)$ with natural and Doppler broadening functions,

$$
i(\nu)=h(\nu) \otimes l(\nu) \otimes d(\nu) .
$$

The hyperfine splitting $h(\nu)$ is a series of $n$ Dirac delta functions,

$$
h(\nu)=\sum_{j=1}^{n} p_{j} \delta\left(\nu-\nu_{j}\right) .
$$

where $\nu_{j}$ is the $j^{\text {th }}$ hyperfine line center and $p_{j}$ is the $j^{\text {th }}$ hyperfine line intensity. The $5 d^{4} D_{7 / 2} \rightarrow 6 p^{4} P_{5 / 2}^{0}$ and $5 d^{2} F_{7 / 2} \rightarrow 6 p^{2} D_{5 / 2}^{0}$ transitions of Xe II has $n=19$ such hyperfine splittings.

Hyperfine structure consists of two components. Isotopic splitting is caused by the mass and nuclear volume differences between isotopes in the sample. Nuclear-spin splitting is caused by the coupling between the nuclear spin $\mathbf{I}$ and the total electronic angular momentum $\mathbf{J}$. For the sake of brevity, we will henceforth use the term "HFS constants" to denote the combined set of isotopic shifts and nuclear-spin interaction constants.

There are nine stable isotopes of xenon, seven of which have natural abundances greater than one percent. Each of these isotopes causes a slightly different transition energy $E_{i j}$, resulting in isotopic splitting. Both of the computational models in this study use naturally-occurring xenon isotopic abundances ${ }^{15}$ while we take isotopic shifts (conventionally defined relative to the center frequency for ${ }^{132} \mathrm{Xe}$ ) for the $5 d^{4} D_{7 / 2}-6 p^{4} P_{5 / 2}^{0}$ transition at $605.1 \mathrm{~nm}$ from the fast ion-beam LIF surveys of Bingham et al. ${ }^{16}$ and Borghs et al. ${ }^{17}$

Seven of the nine stable isotopes of xenon have even atomic mass, resulting in no nuclear spin $\mathbf{I}$. The two isotopes with an odd atomic mass, however, have non-zero nuclear spin quantum numbers $I$. The lighter isotope, ${ }^{129} \mathrm{Xe}$, has $I=1 / 2$, while ${ }^{131} \mathrm{Xe}$ has $I=3 / 2$. These non-zero nuclear spins cause nuclear-spin splitting of the atomic energy levels. This nuclear-spin splitting is 1 to 2 orders of magnitude broader than the isotopic splitting, and provides most of the characteristic shape of the $5 d^{4} D_{7 / 2}-6 p{ }^{4} P_{5 / 2}^{0}$ line at 605.1 nm. The $5 d^{2} F_{7 / 2} \rightarrow 6 p^{2} D_{5 / 2}^{0}$ line at $834.7 \mathrm{~nm}$ is considerably narrower, but nuclear-spin splitting also has a disproportionate influence on the lineshape.

The total angular momentum $\mathbf{F}=\mathbf{I}+\mathbf{J}$ takes quantum number values

$$
F=I+J, I+J-1, \ldots,|I-J|
$$

where $J$ is the total electronic angular momentum quantum number. ${ }^{18}$ The extra term energy due to nuclear-spin splitting is given by ${ }^{19}$

$$
E_{\mathrm{nss}}=A \frac{C}{2}+B D
$$


where $A$ is the nuclear magnetic dipole interaction constant, $B$ is the nuclear electric quadrupole interaction constant, and the terms

$$
C=F(F+1)-I(I+1)-J(J+1)
$$

and

$$
D=\frac{(3 C / 4)(C+1)-I(I+1) J(J+1)}{2 I(2 I-1) J(2 J-1)}
$$

contain the nuclear spin-orbit interactions. Table 1 presents Bröstrom's nuclear-spin structure constants for the Xe II $5 d^{4} D_{7 / 2}, 6 p^{4} P_{5 / 2}^{0}$ and $6 p^{2} D_{5 / 2}^{0}$ energy levels. ${ }^{20}$

Table 1. Published nuclear-spin structure constants for the Xe II transitions at $605.1 \mathrm{~nm}$ and $834.7 \mathrm{~nm}$.

\begin{tabular}{lccc}
\hline \hline Level & $\begin{array}{c}A_{129} \\
(\mathrm{MHz})\end{array}$ & $\begin{array}{c}A_{131} \\
(\mathrm{MHz})\end{array}$ & $\begin{array}{c}B_{131} \\
(\mathrm{MHz})\end{array}$ \\
\hline $5 d^{4} D_{7 / 2}$ & $-502 \pm 4$ & $150 \pm 2$ & $70 \pm 13$ \\
$6 p^{4} P_{5 / 2}^{0}$ & $-1633 \pm 11$ & $487 \pm 4$ & $-129 \pm 16$ \\
$6 p^{2} D_{5 / 2}^{0}$ & $-1387 \pm 9$ & $409 \pm 2$ & $-117 \pm 11$ \\
\hline \hline
\end{tabular}

The transition rule for nuclear-spin splitting is $\triangle F \equiv F-F^{\prime}=[0, \pm 1]$, where $F$ is the upper and $F^{\prime}$ is the lower state's total angular momentum quantum number. (The zero-zero transition is forbidden, $F=0 \nrightarrow F^{\prime}=0$.) Since the total electronic angular momentum quantum numbers are the same for both transitions $\left(J=7 / 2 \rightarrow J^{\prime}=5 / 2\right)$, both lines have the same range of allowable total angular momentum quantum numbers. As a result, Figure 1 shows the fine structure and nuclear-spin splitting for both the $5 d^{4} D_{7 / 2}-6 p^{4} P_{5 / 2}^{0}$ and the $5 d^{2} F_{7 / 2} \rightarrow 6 p^{2} D_{5 / 2}^{0}$ lines.

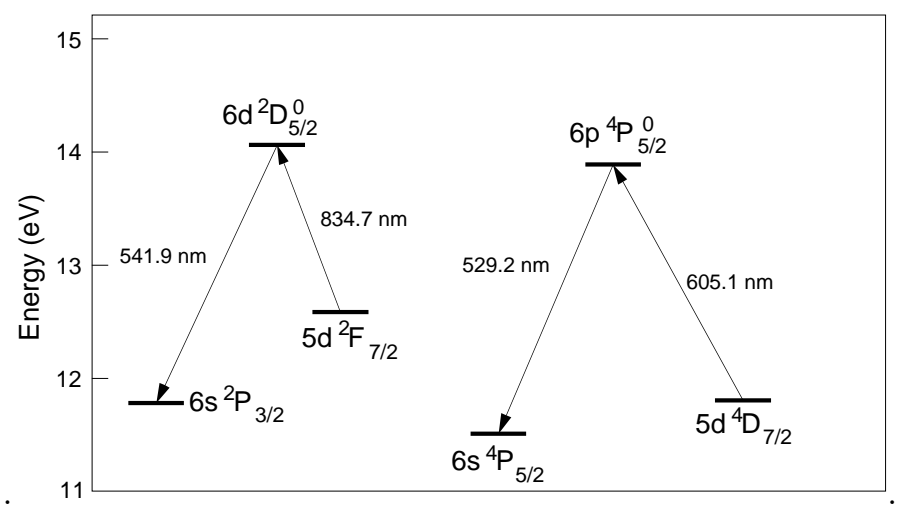

(a) Fine structure.

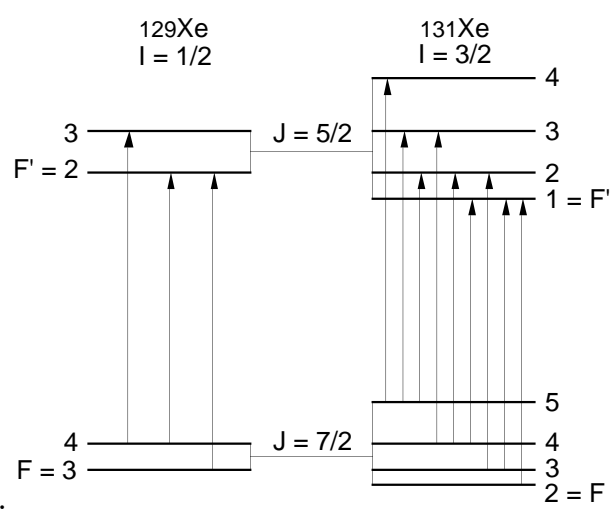

(b) Hyperfine structure for absorption lines.

Figure 1. Fine and hyperfine structure for $605.1 \mathrm{~nm}$ and $834.7 \mathrm{~nm}$ Xe II transitions.

The relative intensity of each nuclear-spin split component is given for a $J \rightarrow J-1$ transition by ${ }^{21}$

$$
\begin{aligned}
I(F \rightarrow F-1) & \propto \frac{P(F) P(F-1)}{F} \\
I(F \rightarrow F) & \propto \frac{(2 F+1)}{F(F+1)} P(F) Q(F) \\
I(F-1 \rightarrow F) & \propto \frac{Q(F) Q(F-1)}{F}
\end{aligned}
$$

where $P(F)=(F+J)(F+J+1)-I(I+1)$ and $Q(F)=I(I+1)-(F-J)(F-J+1)$.

For the isotopes with even mass numbers (i.e., without nuclear-spin splitting), the line intensity $p_{j}$ is linearly proportional to the naturally-occurring abundance for each isotope. The line intensities of ${ }^{129} \mathrm{Xe}$ 
and ${ }^{131} \mathrm{Xe}$ are linearly proportional to the product of the isotopic abundance and the relative intensity of the nuclear-spin split components. It is worth noting that the relative intensity is not a function of the HFS constants, but is the same for both the $5 d^{4} D_{7 / 2}-6 p{ }^{4} P_{5 / 2}^{0}$ and the $5 d^{2} F_{7 / 2} \rightarrow 6 p{ }^{2} D_{5 / 2}^{0}$ lines. This fact considerably simplifies the task of estimating HFS constants from the $834.7 \mathrm{~nm}$ data.

Table 2 presents naturally-occurring xenon isotopic abundances and shifts for the $5 d^{4} D_{7 / 2}-6 p{ }^{4} P_{5 / 2}^{0}$ transition taken from the fast ion-beam LIF surveys of Bingham et al. ${ }^{16}$ and Borghs et al. ${ }^{17}$

Table 2. Isotopic abundance and shift relative to ${ }^{132} \mathrm{Xe}$ for the $5 d^{4} D_{7 / 2}-6 p{ }^{4} P_{5 / 2}^{0}$ transition at $605.1 \mathrm{~nm}$.

\begin{tabular}{l|ccccccccc}
\hline \hline Mass (amu) & 124 & 126 & 128 & 129 & 130 & 131 & 132 & 134 & 136 \\
Abundance (\%) & 0.0096 & 0.009 & 1.92 & 26.4 & 4.1 & 21.1 & 26.9 & 10.4 & 8.9 \\
Shift (MHz) & 336.6 & 252.4 & 172.0 & 113.7 & 83.6 & 16.7 & 0.0 & -75.8 & -140.9 \\
\hline \hline
\end{tabular}

\section{Deconvolution}

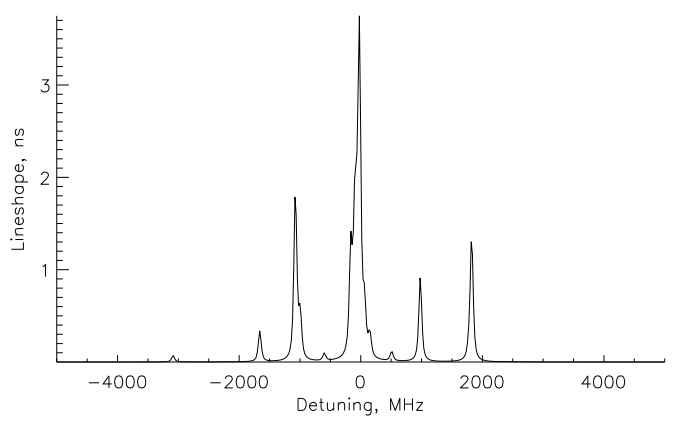

(a) Cold-plasma spectrum $c(\nu)$.

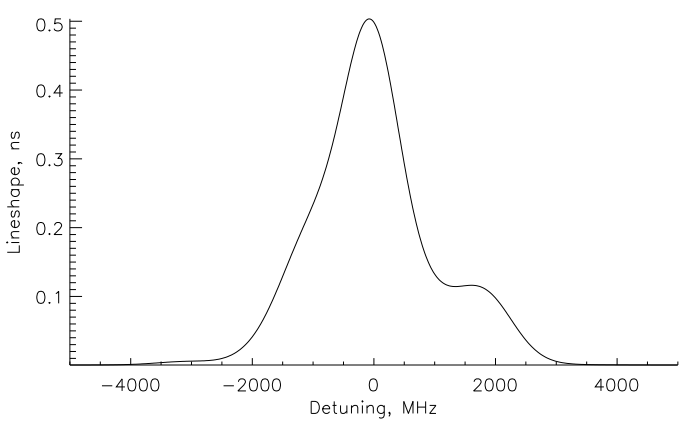

(b) Warm-plasma spectrum $w(\nu), T=1255 \mathrm{~K}$.

Figure 2. Cold-plasma and warm-plasma spectra for the Xe II $5 d^{4} D_{7 / 2}-6 p^{4} P_{5 / 2}^{0}$ line at $605.1 \mathrm{~nm}$.

Figure 2(a) shows the $605.1 \mathrm{~nm}$ absorption spectrum for a perfectly cold stationary plasma, where the velocity distribution $f(\mathbf{v})=\delta(\mathbf{v})$. This can be described by the convolution

$$
c(\nu)=h(\nu) \otimes l(\nu)
$$

so that Eqn. 10 becomes (in the absence of noise)

$$
w(\nu)=c(\nu) \otimes d(\nu) .
$$

Given the Fourier transforms $C(\tau)$ and $D(\tau)$ of the cold-plasma and Doppler broadening functions $c(\nu)$ and $d(\nu)$, the convolution theorem states that Eqn. 20 is equivalent to the product

$$
I(\tau)=C(\tau) D(\tau)
$$

where $I(\tau)$ is the Fourier transform of $i(\nu)$. It then follows that the Doppler broadening transform estimate

$$
\hat{D}_{s}(\tau)=I(\tau) / C(\tau)
$$

is equivalent to the deconvolution

$$
\hat{d}_{s}(\nu)=i(\nu) \oslash c(\nu)
$$

which can be converted to a beamwise velocity distribution estimate by the transformation

$$
\hat{f}\left(v_{k}\right)=\frac{1}{\lambda_{0}} \hat{d}_{s}\left(\left[1-\frac{v_{k}}{c}\right] \nu_{0}\right)
$$


This simple inverse filter approach, though theoretically sound, quickly collapses for realistic signal-tonoise ratios. Deconvolution methods tend to preferentially amplify high-frequency noise. ${ }^{22}$ Low-pass filtering of the deconvolved velocity distribution estimate $\hat{f}\left(v_{k}\right)$ with a Gaussian function of bandwidth $\mathcal{T}$ yields an improved Doppler broadening transform estimate

$$
\hat{D}_{g}(\tau)=\frac{\exp \left(-[\tau / \mathcal{T}]^{2}\right)}{C(\tau)} I(\tau)
$$

that is equivalent to convolving the simple Doppler broadening function estimate with a scale-similar Gaussian,

$$
\hat{d}_{g}(\nu)=\pi \mathcal{T}^{2} \exp \left(-[\pi \mathcal{T} \nu]^{2}\right) \otimes \hat{d}_{s}(\nu)
$$

We have found ${ }^{23}$ that a bandwidth of $2 \mathrm{~ns} \leq \mathcal{T} \leq 3$ ns reduces the noise amplification factor to an acceptable level for LIF and OGS spectra with reasonable signal-to-noise ratios $(S / N \geq 30)$, at the cost of discarding some high-frequency information along with the noise.

\section{Conjugate-gradient method}

Deconvolution of the velocity distribution from LIF and OGS spectra requires a solid model of the coldplasma spectrum $c(\nu)$ for the absorption line. In the absence of published isotopic shifts and lower-state nuclear-spin constants for the $5 d^{2} F_{7 / 2} \rightarrow 6 p^{2} D_{5 / 2}^{0}$ transition at $834.7 \mathrm{~nm}$, we used the conjugate gradient (CG) method ${ }^{25}$ to recover the HFS constants from OGS spectra.

The CG method is an iterative procedure that recovers a local extremum of the function of interest $e(\vec{x})$ by successive searches along steepest descent directions in the parameter space denoted by $\vec{x}$. Consider the absorption line model $w\left(\vec{x}, \nu_{j}\right)$, where the parameter space

$$
\vec{x}=\left[A_{129}, A_{131}, B_{131}, \triangle \nu_{128}, \triangle \nu_{129}, \triangle \nu_{130}, \triangle \nu_{131}, \triangle \nu_{134}, \triangle \nu_{136}\right]
$$

includes the two lower-state nuclear magnetic dipole interaction constants $\left(A_{129}\right.$ and $\left.A_{131}\right)$, the one lowerstate nuclear electric quadrupole interaction constant $\left(B_{131}\right)$, and six isotopic shifts $\left(\triangle \nu_{128}, \triangle \nu_{129}, \triangle \nu_{130}\right.$, $\triangle \nu_{131}, \triangle \nu_{134}$, and $\left.\triangle \nu_{136}\right)$. In the optimization problem of searching for the $834.7 \mathrm{~nm}$ HFS constants contained in $\vec{x}$, we want to minimize the error function

$$
e(\vec{x})=\sum_{j=1}^{n}\left[i\left(\nu_{j}\right)-w\left(\vec{x}, \nu_{j}\right)\right]^{2}
$$

where the measured OGS spectrum $i(\nu)$ consists of $n$ measurements of the OG cell current $i\left(\nu_{j}\right)$ at $n$ frequency shifts $\nu_{j}$.

The first step of the procedure is a guess of the solution, $\vec{x}_{0}$. This initial guess must be reasonably close to the target solution, or else the method may return a solution that corresponds to a local minimum, rather than the desired global minimum. We found that a good initial guess could found from the intensity-weighted nuclear-spin splitting magnitudes

$$
\begin{aligned}
\theta_{129}^{2} & =\frac{1}{3} \sum_{i=1}^{3}\left(p_{i} \triangle \nu_{i}\right)^{2} \\
\text { and } \theta_{131}^{2} & =\frac{1}{9} \sum_{i=1}^{9}\left(p_{i} \triangle \nu_{i}\right)^{2}
\end{aligned}
$$

where $p_{i}$ is the relative intensity of the hyperfine line and $\Delta \nu_{i}=\left(E_{\mathrm{nss}}^{\prime}-E_{\mathrm{nss}}\right) / h$ is the hyperfine line shift. Since the $834.7 \mathrm{~nm}$ line shows much less hyperfine broadening than the $605.1 \mathrm{~nm}$ line, we used HFS constants that minimize $\theta_{129}\left(A_{129}\right)$ and $\theta_{131}^{2}\left(A_{131}, B_{131}\right)$ to specify $\vec{x}_{0}$.

The initial steepest-descent vector $\vec{d}_{0}$ from $\vec{x}_{0}$ is the negative of the gradient of the error function at $\vec{x}_{0}$,

$$
\overrightarrow{d_{0}}=-\nabla e\left(\vec{x}_{0}\right) .
$$

Note that $\vec{d}_{0}$ is not necessarily a vector pointing towards the desired minimum. We can only expect that there exists some local minimum $\vec{x}_{m, 0}$ along the intersection defined by a hyper-plane $\mathbf{P}_{0}$ containing $\vec{d}_{0}$ and 
the hyper-surface $e(\vec{x})$ defining our error function. The minimum along this first hyperplane, which becomes the second guess at the solution $\vec{x}_{1}$, is given by

$$
\vec{x}_{m, 0}=\vec{x}_{0}+\alpha_{0} \vec{d}_{0}=\vec{x}_{1}
$$

where the coefficient $\alpha_{0}$ is an optimal displacement length found by the requirement that the new steepestdescent vector $\vec{d}_{1}$ must be orthogonal to the initial gradient,

$$
-\nabla e\left(\overrightarrow{x_{1}}\right) \cdot \overrightarrow{d_{0}}=0 .
$$

Substitituing Eq. 32 into Eq. 33 yields the condition

$$
-\nabla e\left(\vec{x}_{0}+\alpha \overrightarrow{d_{0}}\right) \cdot \vec{d}_{0}=0 .
$$

This, and the optimal displacement lengths corresponding to subsequent steepest-descent vectors, can be iteratively solved via the secant method, ${ }^{25}$

$$
\begin{aligned}
\alpha_{i} & =-\sigma_{i} \frac{\nabla e\left(\vec{x}_{i}\right) \cdot \vec{d}_{i}}{\nabla e\left(\vec{x}_{i}+\sigma_{i}\right) \cdot \vec{d}_{i}-\nabla e\left(\vec{x}_{i}\right) \cdot \vec{d}_{i}} \\
\text { and } \sigma_{i} & =-\alpha_{i} .
\end{aligned}
$$

Convergence on a value of $\alpha_{i}$ by Eq. 36 prompts the calculation of a new steepest-descent vector. In order to avoid retracing prior search paths, we use the Fletcher-Reeves formula ${ }^{24}$

$$
\vec{d}_{i+1}=-\nabla e\left(\vec{x}_{i+1}+\beta_{i+1} \vec{d}_{i}\right.
$$

where the constant

$$
\beta_{i+1}=\frac{\nabla e\left(\vec{x}_{i+1} \cdot \nabla e\left(\vec{x}_{i}\right)\right.}{\nabla e\left(\vec{x}_{i}\right) \cdot \nabla e\left(\vec{x}_{i}\right)}
$$

for $i>0$ and $\beta_{0}=0$.

The primary stopping condition for our CG code is given when the square of the change in $\vec{x}$ drops below a fixed magnitude $\epsilon$,

$$
\left|\vec{x}_{i+1}-\vec{x}_{i}\right|^{2}>\epsilon \text {. }
$$

Given that $\vec{x}_{i+1}=\vec{x}_{i}+\alpha_{i} \vec{d}_{i}$, the stopping condition for convergence becomes

$$
\alpha_{i}^{2} \vec{d}_{i} \cdot \vec{d}_{i}<\epsilon
$$

which requires no a priori knowledge of the next parameter vector $\vec{x}_{i+1}$.

\section{E. Optogalvanic spectroscopy}

Optogalvanic spectroscopy records changes in the current of a constant-voltage discharge as a laser beam passing through the plasma is tuned through an absorption line of one of the constituent plasma species. Since the current in an unmagnetized discharge is dominated by electron flow, the source of the optogalvanic effect is increased electron density due to electron-impact ionization. For singly-ionized xenon, the relevant reaction is

$$
\mathrm{Xe}^{+}+\mathrm{e}^{-} \rightarrow \mathrm{Xe}^{++}+2 \mathrm{e}^{-} .
$$

Since upper states of Xe II have term energies that are closer to the second ionization potential than lower states, they tend to have a higher probability of electron-impact ionization. Thus, absorption increases the upper-state population and depletes the lower-state population; the net change in the electron density is positive, and shows up as an AC component of the galvatron current at the laser chopping frequency.

This AC galvatron current can be modeled as the sum of signal, noise, and background components:

$$
I_{g}(\nu)=I_{o g}\left(\nu, P_{L}\right)+n(\nu)+I_{p}\left(P_{L}\right) .
$$


The optogalvanic current $I_{o g}\left(\nu, P_{L}\right)$, the signal of interest, is a function of the laser frequency $\nu$ and the laser power at the galvatron input window $P_{L}$. Following the semi-empirical method we developed to model the saturated absorption lineshape $i_{s}\left(\nu, P_{L}\right),{ }^{23}$ the optogalvanic current can be modeled by

$$
I_{o g}\left(\nu, P_{L}\right) \propto \frac{\beta P_{L} i(\nu)}{1+\beta P_{L} i(\nu)}
$$

where $i(\nu)$ is the unsaturated absorption lineshape and the saturation coefficient $\beta$ is a experimentallydetermined constant for a given transition and set of plasma parameters.

Though the noise component $n(\nu)$ can be diminished by ensemble averaging of multiple scans, we have found that there is an irreducible background current $I_{p}\left(P_{L}\right)$ for any optogalvanic setup. This suggests that there is another source of increased discharge current at the chopping frequency. Good centering of the laser beam through the galvatron electrodes tends to increase the signal-to-background ratio, as does reduction of the beam diameter via improved focus and/or a field stop.

We believe that the background current $I_{p}\left(P_{L}\right)$ can be explained as photoelectric emission, induced by the absorption at the cathode surface of laser scattering and Fresnel reflections within the galvatron. This scales linearly with the photon flux to the cathode surface, $I_{p}\left(P_{L}\right) \propto P_{L}$; thus, for a given experimental setup, the maximum signal-to-background ratio becomes

$$
\frac{I_{o g}}{I_{p}} \propto \frac{\beta \max [i(\nu)]}{1+\beta P_{L} \max [i(\nu)]}
$$

which replicates the observed decrease of the signal-to-background ratio with laser power.

\section{Experimental apparatus}

\section{A. Facility}

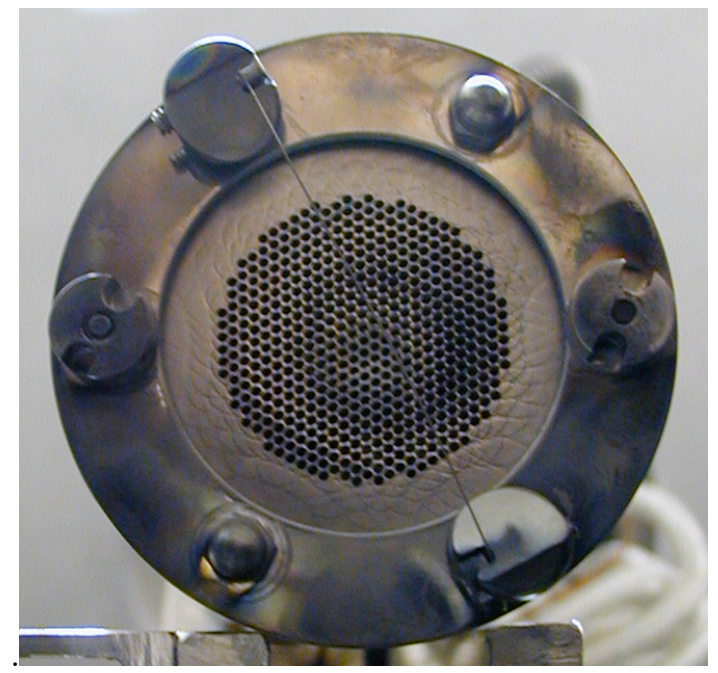

(a) Photograph of the $3 \mathrm{~cm}$ ion source.

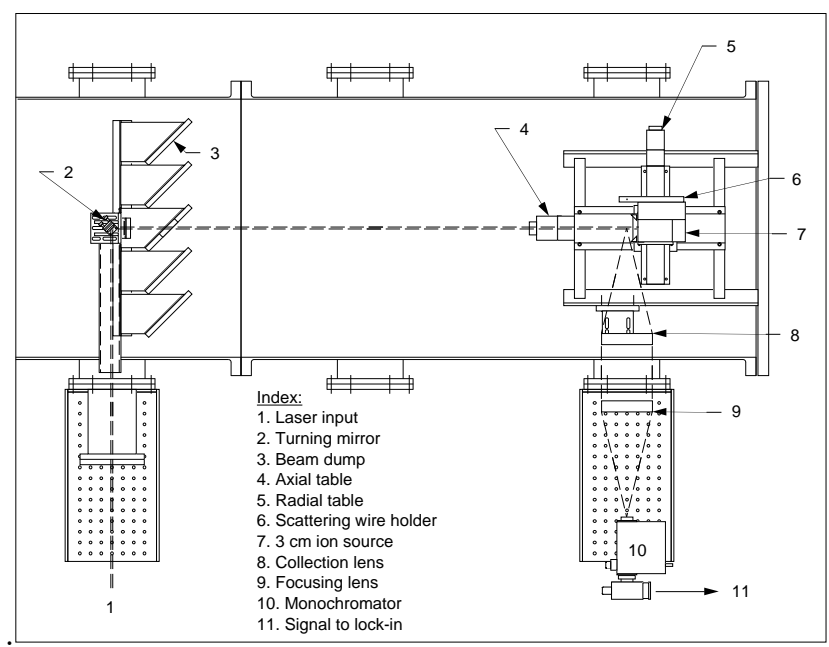

(b) Beam-handling and collection optics, plan view.

Figure 3. Experimental setup in the Cathode Test Facility.

All LIF experiments were carried out in the Cathode Test Facility (CTF) at PEPL. The CTF is a $\phi 0.6 \mathrm{~m}$ $\times 2 \mathrm{~m}$ aluminum-walled cylindrical tank. Ten $\phi 20 \mathrm{~cm}$ flanges allow considerable flexibility in the mounting of optical windows, pressure gauges, feedthroughs, and diagnostics. A five-panel graphite beam dump protects the cryopump and suppresses back sputtering caused by the ion beam.

During operation, an Edwards E2M-30 two-stage mechanical pump roughs the CTF at 135 cfm to approximately 60 mTorr. At this point, the mechanical pumping system is sealed and turned off, and a $\phi 50$ 
cm CVI TM500 cryopump with a measured xenon pumping speed of 1,500 l/s takes over. Base pressure for the facility is $2 \times 10^{-8}$ Torr, as measured with a Varian model 421 cold cathode gauge.

The ion source was mounted on two crossed translation tables, a NEAT TMS-1100-SM single-axis shuttle with 11 inches of travel and a NEAT RMS-800-SM single-axis shuttle with 8 inches of travel. Both translation stages are PC-controlled by a custom LabView VI, with locational resolution on the order of $0.1 \mathrm{~mm}$.

Figure 3(a) shows our Commonwealth Scientific $\phi 3 \mathrm{~cm}$ gridded ion source, a commercial unit capable of operation at up to $1500 \mathrm{~V}$. The outer body of the ion source is a grounded $\phi 63.5 \times 84.8 \mathrm{~mm}$ shell of $1.6 \mathrm{~mm}$ thick stainless steel. The screen and accelerator grids are $\phi 43.5 \times 1 \mathrm{~mm}$ discs with three mounting lugs and a laser-drilled hexagonal pattern of $\phi 1.0 \mathrm{~mm}$ apertures with 1.32 between centers. Alumina spacers (when uncoated by sputtering products) isolate both grids and maintain the grid spacing. A Unit UFC-7301 mass flow controller regulates 0 to $10 \mathrm{sccm}$ of Xe flow into the discharge. A Kikisui PAD 16-10L supply powers the $\phi 0.25 \times 60 \mathrm{~mm}$ tungsten discharge cathode filament, while a Hewlett-Packard HP 6271 supply maintains the discharge current and voltage. Both of these supplies are floated atop a Glassman FC01.5P80 1500-V, 80-mA power supply, while a Sorenson DCS 33-33 supply powers the $\phi 0.25 x 80 \mathrm{~mm}$ neutralizer cathode filament.

\section{B. Lasers and external optics}

The primary laser system used in this experiment, a TUI Optics TA-100/830 tapered-amplifier diode laser, has not previously been used at PEPL. This system has a nominal linewidth of $2 \mathrm{MHz}$, a tuning range of 820 $\mathrm{nm}$ to $836 \mathrm{~nm}$, and a mode-hop-free range of over $10 \mathrm{GHz}$. Though capable of output powers in excess of $500 \mathrm{~mW}$, off-peak efficiency drops and Faraday isolation of the tapered amplifier reduce the available output power at the upper end of the system wavelength range $(835 \pm 1 \mathrm{~nm})$ to approximately $250 \mathrm{~mW}$.

The master oscillator for this system is an ECDL. Coarse wavelength control is provided by Peltier cooling of the ECDL and micrometer adjustment of the ECDL feedback grating angle. PC control of the fine tuning is provided by a LabView VI, which uses the auxiliary output of a Stanford SR810 lock-in amplifier to send a control voltage to a piezoelectric transducer (PZT) driving the ECDL feedback grating. Feedback circuitry in the ECDL power supply decreases the master oscillator diode current during a PZT sweep to maximize the mode-hop-free range. In order to include the effect of changing laser output power, we normalize our reported LIF and OGS spectra by a quadratic curve-fit to the master oscillator power. It is important to note that this normalization carries an implicit assumption that the absorption line is not saturated. Steering mirrors inside the TA-100 enclosure couple the ECDL output into the tapered amplifier diode, which boosts the ECDL output power by a factor of 10 while maintaining the master oscillator wavelength and linewidth.

Figure 4(a) shows a schematic of the diode laser table contents. A wedged beamsplitter sends approximately $5 \%$ of the TA-100 output beam to a steering mirror, and thence to a Burleigh WA-1000 wavemeter. This wavemeter permits wavelength measurement with a $1 \mathrm{pm}$ resolution. Oversampling of the PZT control voltage permits greater wavelength resolution by a quadratic curve-fit to the wavemeter output, but does not improve the absolute bias of the laser wavelength measurement.

The second-surface reflection from the wedged beamsplitter is currently sent to a beam dump. In future experiments, we plan to direct this beam into a recently-refurbished Burleigh SAPlus spectrum analyzer). This $2-\mathrm{GHz}$ etalon, which has a finesse of 256 , will provide repeatable frequency markers at $2-\mathrm{GHz}$ intervals, providing reference markings by which individual $10-\mathrm{GHz}$ scans can be "stitched" together into a reasonably seamless scan over many tens of GHz.

The remaining $90 \%$ of the beam passes through a Stanford SR541 optical chopper, permitting phase-lock amplification of subsequent OGS and LIF signals.

The secondary system, a Coherent 899-29 Autoscan ring dye laser, has been used for LIF measurements for the past six years at PEPL. This PC-controlled system has a nominal linewidth of $500 \mathrm{kHz}$, tuning repeatability of $50 \mathrm{MHz}$ and a scanning range of over $100 \mathrm{GHz}$ (in $10 \mathrm{GHz}$ segments). PC-controlled scanning and data collection are synchronized by the Autoscan software. Pumping for this dye laser is provided by a Coherent SBRC-R-DBW20/4 argon-ion laser, with a nominal broadband power rating of $25 \mathrm{~W}$. With the intercavity assembly (ICA) removed, the dye laser can generate up to $2 \mathrm{~W}$ of tunable broadband light using Rhodamine-6G dye at $605 \mathrm{~nm}$. With the ICA installed, the same system can provide anywhere from 300 to $450 \mathrm{~mW}$ of narrow-linewidth light at $605.1 \mathrm{~nm}$.

In order to combine beams from both laser systems on a single beamline, we designed the beam-mixer shown in Figure 4(b). The heart of this is a polarizing beamsplitter. Run in the usual orientation, it takes a randomly-polarized input beam and outputs two orthogonally-directed beams, each polarized at right angles to one another. In our setup, the beamsplitter takes two orthogonally-polarized input beams and combines 


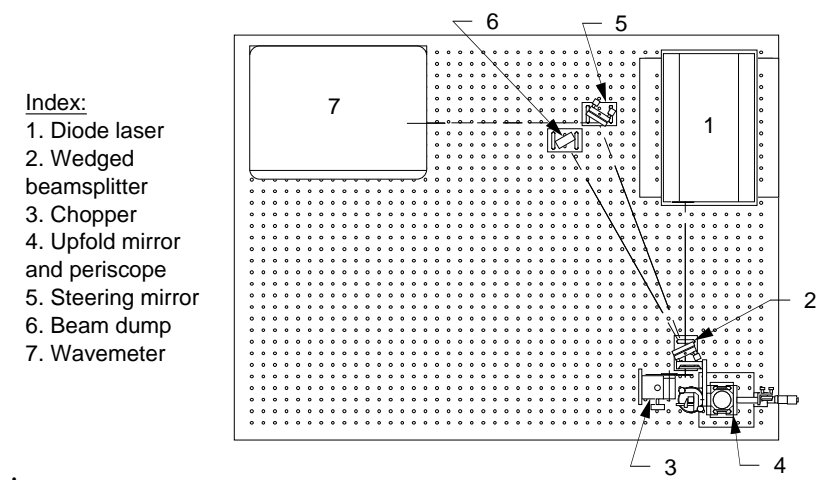

(a) Diode laser table layout, plan view.

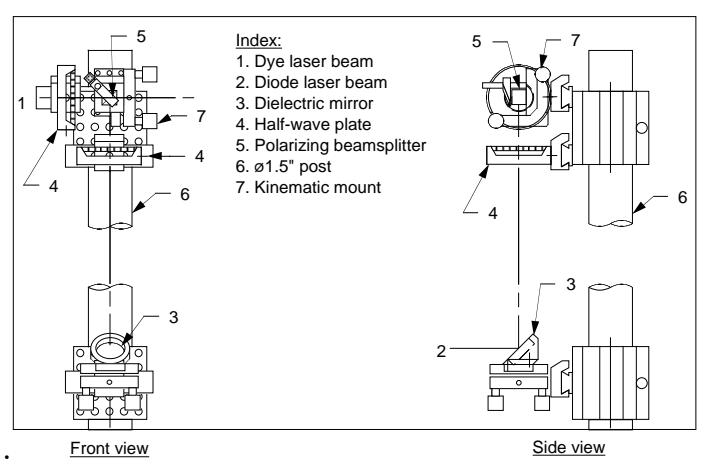

(b) Beam mixer elevations.

Figure 4. Laser beam mixing, modulation and sampling.

them on a single beamline. Half-wave plates, which are mounted in rotation stages between the lasers and the beamsplitter, ensure that the polarization direction of the incoming beams is properly aligned for maximum transmission through the beamsplitter. A kinematic mount permits fine control of the diode laser beam direction, ensuring that the two beams remain overlapped throughout the experimental setup. A $\phi 25 \mathrm{~mm}$, $f=2 \mathrm{~m}$ lens placed at the beam-mixer output places the combined beam focal point within a few $\mathrm{cm}$ of the interrogation volume within the CTF.

Since the dye laser system was not working well enough for reliable LIF during this experiment, we substituted a simple helium-neon laser. Though this is not useful for LIF, it nevertheless provided a valuable tool for visible-light alignment of the laser input and collection optics axes.

Figure 3(b) shows the beam-handling and collection optics setup used for LIF experiments in the CTF. A square $25 \mathrm{~mm}$ mirror on a kinematic mount, attached within an enclosure tube behind the beam dump, redirects the combined laser beams through a $\phi 25 \mathrm{~mm}$ protective window toward the ion source grid. A $\phi 1 \times 80 \mathrm{~mm}$ section of steel music wire, mounted vertically in a tube placed to one side of the ion source, facilitates alignment of the collection optics. After moving the alignment wire into the laser beam path, we adjust the $\phi 100 \mathrm{~mm}, f / 2.5$ collection lens, sending a collimated beam of scattered light through the CTF window. During experiments, the collimated fluorescence follows the same path.

The collimated fluorescence from the ion source plume is focused by a second $\phi 100, f / 2.5$ lens onto the entrance slits of a Spex H-10 monochromator with a Hamamatsu 928 PMT. This monochromator acts as a linewidth filter centered on the $541.9 \mathrm{~nm}$ fluorescence line. By holding a second-surface mirror flat against the monochromator entrance slits, we can use retroreflection of scattered light from the alignment wire to ensure proper alignment of the monochromator with the collection optis axis. The PMT signal is passed to a Stanford SR810 lock-in amplifier, which isolates the fluorescence component of the optical signal entering the PMT.

\section{Optogalvanic cell}

Figure 5 shows the optogalvanic cell setup used on the CTF. A wedged beamsplitter, located just before the CTF input window, sends approximately $4 \%$ of the combined beams to a steering mirror, and thence through the optogalvanic cell. A filter holder placed in the beam path permits neutral density filter attenuation of the incoming laser beam power for saturation studies.

The optogalvanic cell used in this study is a Hamamatsu L2783-42 XeNe-Mo galvatron. The core of the galvatron is a $\phi 6.25 \mathrm{~mm}$ pair of cylindrical Mo electrodes, centered in a $\phi 25 \times 120 \mathrm{~mm}$ glass cylinder filled with approximately 3 Torr of xenon and and 4 Torr of neon. The ends of the cylinder are angled approximately 10 degrees from a perpendicular to the electrode axis; though this is less than Brewsters angle for a glass-air interface, it reduces the likelihood of retroreflected beams entering the discharge. This design permits unobstructed passage of a laser beam along the axis shared by the cylinder and electrodes. 


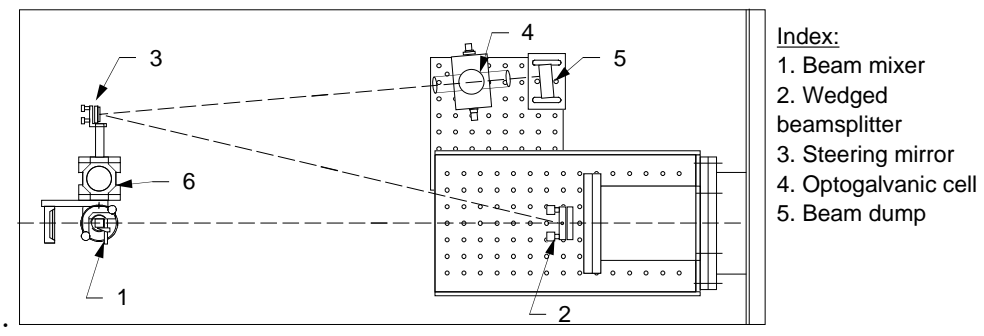

Figure 5. Optogalvanic cell setup on the CTF, plan view.

A $250 \mathrm{~V}$ discharge across the galvatron electrodes produces a reasonably cool, dense, stationary plasma. A ballast resistor prevents runaway current growth after the discharge is struck, and provides a simple method of measuring the discharge current. The voltage drop across the ballast resistor is connected to the Stanford SR-810 lock-in amplifier through a $3.33 \mu \mathrm{F}, 450 \mathrm{~V}$ capacitor. The capacitor passes the AC component of the voltage drop, while isolating the lock-in from high DC voltages.

\section{Retarding potential analyzer}

The RPA used in this experiment is based on the three-grid AFRL design described by Beal. ${ }^{26}$ The outer body of the RPA is a grounded $\phi 38 \times 32 \mathrm{~mm}$ shell of $1 \mathrm{~mm}$ thick 316 stainless steel. A phenolic sleeve isolates the grids, which are identical $\phi 32 \times 0.125 \mathrm{~mm}$ disks of 316 stainless steel sheet with an etched hexagonal pattern of $\phi 0.279 \mathrm{~mm}$ apertures. The total open area fraction for each grid is $38 \%$. Macor washers fix the grid separation distances. In a departure from the original AFRL design, the collector is a $\phi 32 \times 1 \mathrm{~mm}$ disk of 316 stainless steel, plasma-sprayed with a tungsten coating to minimize secondary electron emission.

During operation, the outermost grid floats, minimizing perturbations at the probe-plasma interface. A laboratory power supply holds the second (or electron repulsion) grid 30 volts below ground. A Keithley 2410 Sourcemeter (under LabView control through a GPIB interface) then sweeps the third (or ion repulsion) grid potential from 0 to $600 \mathrm{~V}$. A Keithley 6485 picoammeter simultaneously measures and passes the collector current via GPIB to the LabView VI. Numerical differentiation of the collected current as a function of ion retarding grid potential provides an instrument trace that is linearly proportional to the distribution of ion energy per unit charge. For the sake of brevity, we will henceforth refer to this as an ion voltage distribution function. ${ }^{26}$

\section{Results}

\section{A. OGS at $605.1 \mathrm{~nm}$}

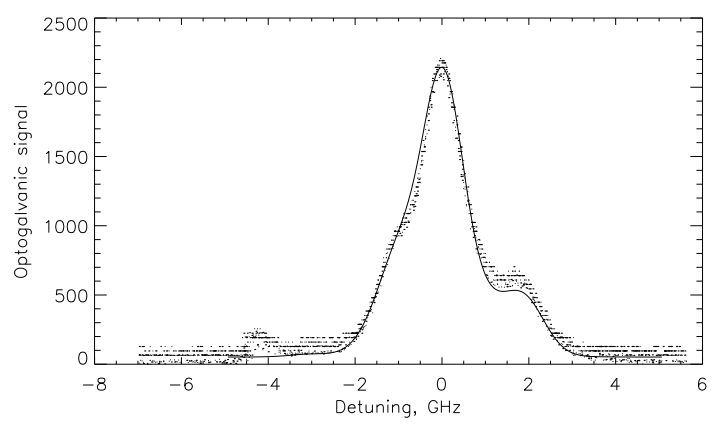

Figure 6. Optogalvanic spectrum at $605.1 \mathrm{~nm}$ (points) and warm-plasma spectrum model, $\mathrm{T}=1255 \mathrm{~K}$ (line).

Figure 6 shows a sample set of seven optogalvanic spectra taken at $605.1 \mathrm{~nm}$ with approximately $8 \%$ 
of the maximum dye laser output. From Maxwellian curve-fitting to velocity distributions deconvolved from 49 such data sets, we estimate that the Xe II temperature in a $250 \mathrm{~V}$ optogalvanic cell discharge is $T=(1255 \pm 57) \mathrm{K}$. The solid line in Figure 6 shows a scaled warm-plasma spectrum model at $T=1255 \mathrm{~K}$, using the published HFS constants and the natural upper-state lifetime (7.8 ns) for the $605.1 \mathrm{~nm}$ absorption line $^{\mathrm{a}}$.

The fit between the model and the data set is generally quite good. The minor peaks in the model tend to fall slightly under the experimental points. This may be evidence of saturation, which tends to preferentially flatten the highest peaks of an absorption line.

The observed signal-to-background ratio for this data set is about 32 . No special efforts were made to minimize the background level during this series of experiments.

\section{B. OGS at $834.7 \mathrm{~nm}$}

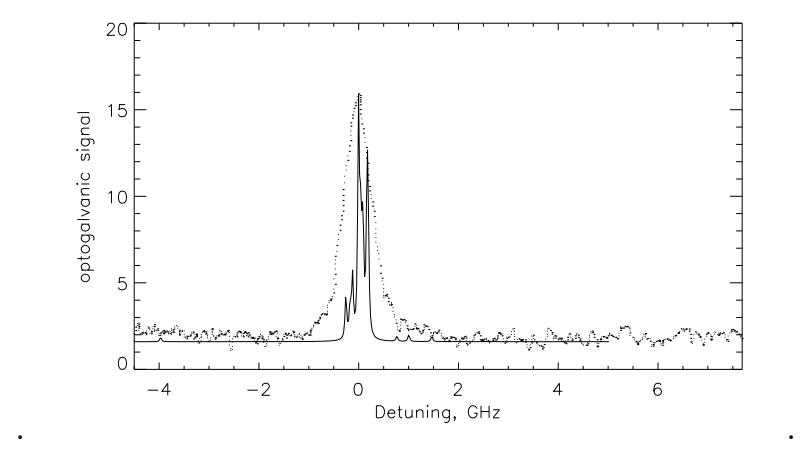

(a) Cold-plasma spectrum $c(\nu)$

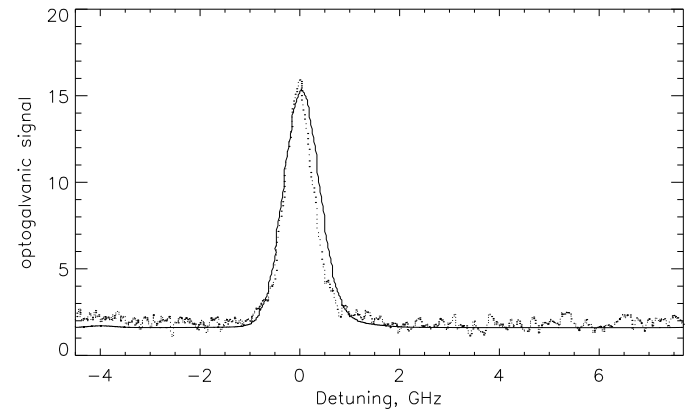

(b) Warm-plasma spectrum $w(\nu), \mathrm{T}=1255$

Figure 7. Optogalvanic spectrum at $834.7 \mathrm{~nm}$ : data (points) and spectrum model (line).

Figure 7 shows the ensemble average of 64 optogalvanic spectra taken at $834.7 \mathrm{~nm}$ with a $110 \mu \mathrm{W}$ diode laser beam at the galvatron entrance. We took especial pains to minimize the photoelectric effect for this data set, yielding a modest signal-to-background ratio of about 8. Beam quality (i.e. the level of departure from a perfect Gaussian beam profile), which is generally far worse for diode lasers than dye lasers, may be one culprit; however, placing a field stop in front of the galvatron did not yield impressive improvements in the signal-to-background level. The relative difficulty in achieving a good signal-to-background ratio at $834.7 \mathrm{~nm}$, when compared to the ease with which we achieved far better results at $605.1 \mathrm{~nm}$, suggests that the optogalvanic effect at $834.7 \mathrm{~nm}$ is relatively weak. Since both lines exhibit strong emission in discharge plasmas, the weak optogalvanic effect implies that the difference in upper-state and lower-state ionization probabilities is far lower for the $834.7 \mathrm{~nm}$ line than the $605.1 \mathrm{~nm}$ line.

This ensemble average served as the experimental data set for conjugate-gradient optimization of the computational model. Figure 7(a) overlays the data set with the best-fit cold-plasma spectrum, using the published natural upper-state $(9.3 \mathrm{~ns})$ to set the Lorentzian linewidth. Figure 7(b) overlays the same data set with a best-fit warm-plasma spectrum, using the ion temperature calculated from the $605.1 \mathrm{~nm}$ data to set the Doppler broadening.

The fit between the model and the data set is not as good as the fit in Figure 6. The model is uniformly broader than the data set. One possible explanation for this excessive broadening is an error in the broadening model. Our knowledge of the linewidths for broadening mechanisms can be impugned; not only is the galvatron pressure far higher than typically acceptable for purely natural broadening, but the saturation effects noted in the $605.1 \mathrm{~nm}$ optogalvanic spectra may exaggerate the reference cell temperature. Unfortunately, we currently have insufficient information to determine the validity of either hypothesis; further validation from the existing data set must rest on how well ion energy distributions deconvolved from 834.7 nm LIF data compare with RPA data.

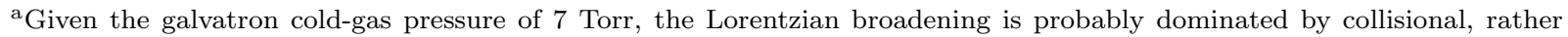
than radiative, effects. In the absence of better information, we have opted to use the natural linewidth for this study. 
Table 3 compares the previously-described "first guess" $\vec{x}_{0}$ with the final set of isotopic shifts and lowerstate $\left(5 d^{2} F_{7 / 2}\right)$ nuclear-spin constants returned by conjugate-gradient optimization. The similarity between the two sets of constants is striking; with the current data set, the simple expedient of choosing lowerstate nuclear-spin constants to minimize the hyperfine-broadened linewidth comes very near to an optimal estimate. The error function for the first-guess set of constants is only $0.87 \%$ worse than the final estimate! Subsequent reconvergence studies in which we varied the Xe II temperature by the standard deviation (55 $\mathrm{K}$ ) of the $605.1 \mathrm{~nm}$ temperatures resulted in isotopic shift and nuclear-spin constant uncertainties on the order of $15 \mathrm{MHz}$.

Table 3. Isotopic shifts and $5 d^{2} F_{7 / 2}$ nuclear-spin constants from $834.7 \mathrm{~nm}$ optogalvanic spectra

\begin{tabular}{cccccccccc}
\hline \hline \multirow{2}{*}{$\begin{array}{c}A_{129} \\
\text { Solution }\end{array}$} & $\begin{array}{c}A_{131} \\
(\mathrm{MHz})\end{array}$ & $\begin{array}{c}B_{131} \\
(\mathrm{MHz})\end{array}$ & $\begin{array}{c}\triangle \nu_{128} \\
(\mathrm{MHz})\end{array}$ & $\begin{array}{c}\triangle \nu_{129} \\
(\mathrm{MHz})\end{array}$ & $\begin{array}{c}\triangle \nu_{130} \\
(\mathrm{MHz})\end{array}$ & $\begin{array}{c}\triangle \nu_{131} \\
(\mathrm{MHz})\end{array}$ & $\begin{array}{c}\triangle \nu_{134} \\
(\mathrm{MHz})\end{array}$ & $\begin{array}{c}\triangle \nu_{136} \\
(\mathrm{MHz})\end{array}$ \\
\hline First guess & -1030.5 & 297.2 & 13.2 & 170.73 & 92.379 & 73.461 & -17.581 & 86.655 & 43.282 \\
Convergence & -1035.4 & 316.3 & 14.33 & 171.25 & 92.97 & 74.257 & -19.225 & 89.753 & 44.747 \\
\hline \hline
\end{tabular}

\section{LIF velocimetry}

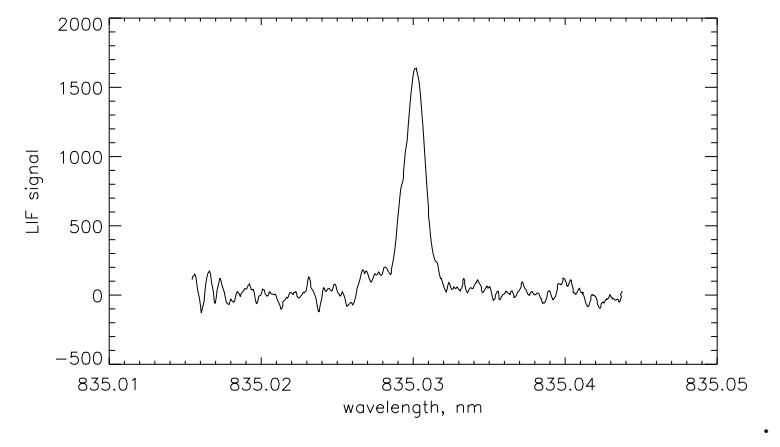

(a) LIF spectrum.

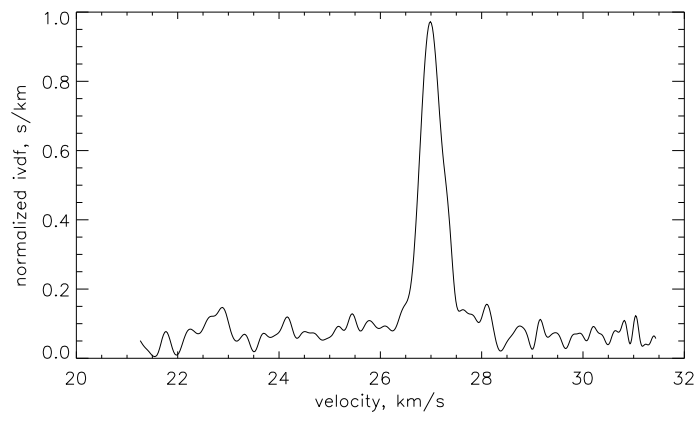

(b) Ion velocity distribution from LIF.

Figure 8. LIF spectrum and ion velocity distribution at $834.7 \mathrm{~nm}$ in a $500 \mathrm{~V}, 30 \mathrm{~mA}$ ion beam.

Figure 8(a) shows the ensemble average of $62 \mathrm{LIF}$ spectra taken at $834.7 \mathrm{~nm}$ in the $500 \mathrm{~V}$ ion source plume. Each 120-second scan consists of 501 data points taken with a lock-in time constant of $300 \mathrm{~ms}$. After transmission losses, the diode laser power entering the interrogation volume dropped from $47.2 \mathrm{~mW}$ (at the high- $\lambda$ end of the scan) to $24.7 \mathrm{~mW}$ (at the low- $\lambda$ end of the scan). During these tests, we set an anode potential of $500 \mathrm{~V}$, a discharge voltage of $32.0 \mathrm{~V}$, an accel grid potential of $-75.0 \mathrm{~V}$, and a xenon volume flow rate of $2.0 \mathrm{sccm}$. Manual adjustment of the cathode filament current maintained the beam current at $30.0 \pm 0.3 \mathrm{~mA}$. Charge neutralization in the beam was ensured by increasing the neutralizer filament current until neutralization arcs were no longer observed.

Over the course of this test, the discharge current required to maintain the beam current at $30 \mathrm{~mA}$ dropped unsteadily from $638 \mathrm{~mA}$ to $145 \mathrm{~mA}$. Isolation checks showed that the accelerator grid isolation dropped from an unreadably high pre-test value to a post-test value of $50.0 \mathrm{k} \Omega$. Post-test examination of the ion source showed severe damage to the accelerator grid. A new hexagonal set of $\phi 0.75 \mathrm{~mm}$ apertures, offset approximately $0.75 \mathrm{~mm}$ from the original grid apertures, had been etched through the accelerator grid. We believe this to be caused by misalignment of the grids during reassembly, as the isolators had just been removed for cleaning before the test.

Figure 8(b) shows the ion velocity distribution deconvolved from these LIF spectra. The deconvolution routine uses the newly-modeled $834.7 \mathrm{~nm}$ cold-plasma spectrum, a Gaussian inverse filter with a $2.0 \mathrm{~ns}$ 
bandwidth, and the optogalvanic spectrum peak of $834.955 \mathrm{~nm}$ (vacuum) as the stationary plasma reference wavelength. The mode ion speed for this distribution is $26.985 \mathrm{~km} / \mathrm{s}$, while the mean ion speed for the primary peak (defined as the area between the two minima on either side of the ivdf maximum) is $\langle v\rangle=26.890 \mathrm{~km} / \mathrm{s}$. The central moments of the principal peak, defined as

$$
u^{(k)}=\left\langle(v-\langle v\rangle)^{k}\right\rangle,
$$

are $u^{(2)}=4.6132(\mathrm{~km} / \mathrm{s})^{2}, u^{(3)}=-2.7830(\mathrm{~km} / \mathrm{s})^{3}$, and $u^{(4)}=67.507(\mathrm{~km} / \mathrm{s})^{4}$. These moments would equal zero for a pure Maxwellian distribution; for the measured distribution, they indicate that the peak is leftskewed $\left(u^{(3)}<0\right)$ and has mild leptokurtosis $\left(u^{(4)}>0\right)$. Since $u^{(2)}=k T / M$ for a Maxwellian distribution, the equivalent temperature for the primary peak is an astonishingly large $6.28 \mathrm{eV}$.

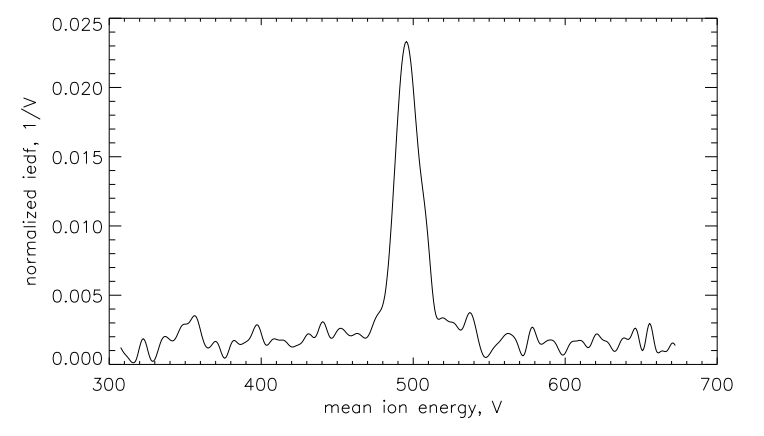

(a) Ion energy distribution from LIF.

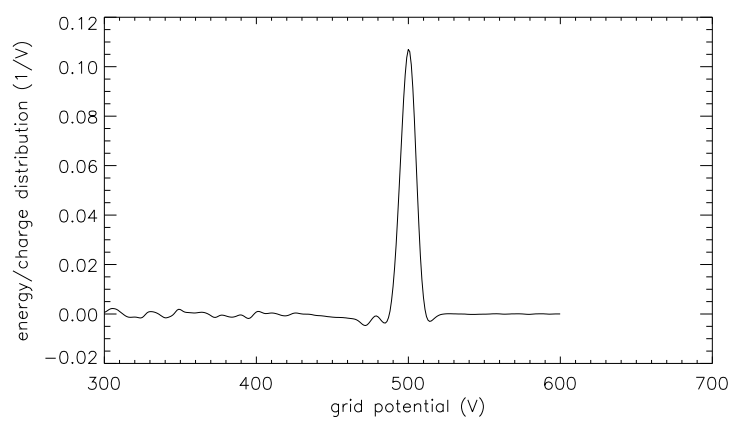

(b) Ion voltage distribution from RPA.

Figure 9. LIF and RPA ion energy distributions in a $500 \mathrm{~V}$ ion beam.

Figure 9(a) shows the same distribution converted to an ion energy basis, while Figure 9(b) shows the ion voltage distribution from previous RPA testing in a $500 \mathrm{~V}$ ion beam. The ion-retarding grid potential was raised in $1-\mathrm{V}$ steps during this scan, while the electron-retarding grid potential was held at a constant $30 \mathrm{~V}$ below ground. During the RPA test, we set an anode potential of $500 \mathrm{~V}$, a discharge voltage of 32.0 $\mathrm{V}$, an accel grid potential of $-75.0 \mathrm{~V}$, and a xenon volume flow rate of $3.0 \mathrm{sccm}$. Manual adjustment of the cathode filament current maintained the beam current at $30.0 \pm 0.3 \mathrm{~mA}$. Charge neutralization in the beam was ensured by increasing the neutralizer filament current until neutralization arcs were no longer observed. Over the course of the test, the discharge current required to maintain this beam current remained steady.

Table 4. Central moments of the ion energy distribution function main peak at $500 \mathrm{~V}$.

\begin{tabular}{lccccc}
\hline \hline & $\begin{array}{c}\text { mode } E \\
(\mathrm{~V})\end{array}$ & $\begin{array}{c}E^{(1)} \\
(\mathrm{V})\end{array}$ & $\begin{array}{c}E^{(2)} \\
\left(\mathrm{V}^{2}\right)\end{array}$ & $\begin{array}{c}E^{(3)} \\
\left(\mathrm{V}^{3}\right)\end{array}$ & $\begin{array}{c}E^{(4)} \\
\left(\mathrm{V}^{4}\right)\end{array}$ \\
\hline $\mathrm{LIF}$ & 495.45 & 495.10 & 6059.4 & -12884. & $1.1389 \times 10^{8}$ \\
$\mathrm{RPA}$ & 500. & 499.88 & 18.957 & -5.8123 & 879.82 \\
\hline \hline
\end{tabular}

Table 4 compares mode energies and the central moments of the principal peak for both distributions. As before, we define the $\mathrm{k}^{\text {th }}$ central moment of the energy distribution as

$$
E^{(k)}=\left\langle(E-\langle E\rangle)^{k}\right\rangle
$$

The mode and mean energies for both measurements agree within 1\%, and the third moments show that both distributions are left-skewed $\left(E^{(3)}<0\right)$. Otherwise, though, the higher moments point out that the LIF distribution is far wider and has much wider tails than the RPA distribution. As mentioned above, the equivalent temperature for an equally wide Maxwellian is $6.28 \mathrm{eV}$, which is at least an order of magnitude hotter than the axial Xe II temperatures we have previously measured in the P5 Hall thruster plume with $605.1 \mathrm{~nm} \mathrm{LIF.}^{1}$ 
We believe (but can not yet prove) that the aforementioned grid misalignment is to blame for the unnaturally wide LIF distribution. Heavily-eroded grids lead to electron backstreaming, as indicated by the decrease in discharge current over the course of the test. Both grid misalignment and electron backstreaming tend to increase the beam divergence. Williams ${ }^{27}$ notes that perfectly-cold ions $(f(v)=\delta(v-\langle v\rangle))$ passing through an interrogation volume with a beam divergence angle $\theta$ will have a Doppler-broadened absorption spectrum, with an apparent temperature of

$$
T_{\text {div }}=\frac{1}{2 \ln 2} \frac{M}{k}\left(\frac{\langle v\rangle}{2}\left[1-\cos \frac{\theta}{2}\right]\right)^{2} .
$$

Thus, the divergence angle required for cold ions to produce an observed velocity distribution with the variance $\left\langle(v-\langle v\rangle)^{2}\right\rangle=k T_{\text {div }} / M$ is

$$
\theta=2 \cos ^{-1}\left(1-\frac{2}{\langle v\rangle} \sqrt{2 \ln 2\left\langle(v-\langle v\rangle)^{2}\right\rangle}\right) .
$$

At $\langle v\rangle=26.9 \mathrm{~km} / \mathrm{s}$, a beam divergence angle of $\theta=35.7^{\circ}$ would produce the value of $\left\langle(v-\langle v\rangle)^{2}\right\rangle$ seen in the primary peak of the LIF deconvolution.

\section{Conclusions}

To the best of our knowledge, this study presents the first published set of isotopic shift and lower-state nuclear-spin interaction constant estimates for the $5 d^{2} F_{7 / 2} \rightarrow 6 p^{2} D_{5 / 2}^{0}$ absorption at $834.7 \mathrm{~nm}$. Though the optogalvanic signal-to-background ratio at $834.7 \mathrm{~nm}$ was significantly worse than the signal-to-background ratio for the $5 d^{4} D_{7 / 2} \rightarrow 6 p^{4} P_{5 / 2}^{0}$ absorption at $605.1 \mathrm{~nm}$, we were able to converge on HFS constants that produce an acceptable fit to the observed optogalvanic spectra.

Our attempt to validate these HFS constants by deconvolution of $834.7 \mathrm{~nm}$ LIF spectra was less successful. Aside from the readily-obtained agreement between mean ion energies, the similarities between LIF and RPA ion energy distributions were more qualitative than quantitative. The expected reduction in Doppler broadening from kinematic compression effect did not appear; instead, the LIF velocity distribution was unusually wide. Misalignment of the ion source grids may be responsible for the high width of the LIF velocity distribution.

Future tests of this technique should involve simultaneous LIF and RPA measurements, as a minimum; this would permit early detection of beam divergence effects. A higher-density ion source (such as a Hall thruster) might reduce the extensive integration times required for an acceptable signal-to-noise ratio with a gridded ion source, as would replacing the neutralizer filament with a neutralizing hollow cathode. Ideally, the velocity distribution would be independently and non-intrusively measured by two-wavelength simultaneous LIF (TWSLIF) at 605.1 and $834.7 \mathrm{~nm}$.

\section{Acknowledgements}

This research was supported by the Air Force Office of Scientific Research grants F49620-00-1-0201 and F49620-01-1-0061 (Dr. Mitat Birkan is the contract monitor for both). The authors are greatly appreciative of this support.

We would also like to thank the graduate and undergraduate students at PEPL for their assistance in experimental setup and operation, and the Department's technical support staff for their aid with facility repairs and modifications.

\section{References}

${ }^{1}$ T. B. Smith, D.A. Herman, A.D. Gallimore and R.P. Drake, "Deconvolution of axial velocity distributions from Hall thruster LIF spectra," 27th International Electric Propulsion Conference, IEPC-01-0019, October 2001.

${ }^{2}$ R. J. Cedolin. Laser-induced fluorescence diagnostics of xenon plasmas. Ph.D. dissertation, Dept. of Mechanical Engineering, Stanford University, June 1997.

${ }^{3}$ W. A. Hargus Jr. and M.A. Cappelli, "Interior and exterior laser-induced fluorescence and plasma potential measurements on a laboratory Hall thruster," 35th Joint Propulsion Conference, AIAA-99-2721, June 1999.

${ }^{4}$ W. A. Hargus Jr/ and M. A. Cappelli. "Laser-induced fluorescence measurements of velocity within a Hall discharge." Applied Physics B B72(2001) 961-969. 
${ }^{5}$ W. A. Hargus Jr. Investigation of the plasma acceleration mechanism within a coaxial Hall thruster. Ph.D. dissertation, Dept. of Mechanical Engineering, Stanford University, March 2001.

${ }^{6}$ N. Dorval, J. Bonnet, J. P. Marque and D. Pigache. "Laser induced fluorescence measurements in xenon plasma thrusters." In Proceedings of the 3rd International Conference on Spacecraft Propulsion. ESA-SP-465, Oct 2000, 797-805. Quoted in. ${ }^{7}$

${ }^{7}$ N. Dorval, J. Bonnet, J. P. Marque, E. Rosencher, S. Chable, F. Rogier and P. Lasgorciex. "Determination of the ionization and acceleration zones in a stationary plasma thruster by optical spectroscopy study: Experiments and model." Journal of Applied Physics 91(2002) 4811-4817.

${ }^{8}$ D. H. Manzella. "Stationary plasma thruster ion velocity distribution." In Proceedings of the 30th Joint Propulsion Conference. AIAA-94-3141, June 1994.

${ }^{9}$ E. J. Beiting and J. E. Pollard. "Measurements of xenon ion velocities of the SPT-140 using laser induced fluorescence." In Proceedings of the 3rd International Conference on Spacecraft Propulsion. ESA-SP-465, Oct 2000, 789-797.

${ }^{10}$ J. T. Verdeyen. Laser Electronics. 3rd ed., Prentice-Hall, 1995.

${ }^{11}$ R. Koslover and R. McWilliams. "Measurement of multidimensional ion velocity distributions by optical tomography." Review of Scientific Instruments 57(1986) 2441-2448.

${ }^{12}$ R. A. Dressler, J. P. M. Beijers, H. Meyer, S. M. Penn, V. M. Bierbaum and S. R. Leone. "Laser probing of ion velocity distributions in drift fields: Parallel and perpendicular temperatures and mobility for $\mathrm{Ba}^{+}$in He." Journal of Chemical Physics 89(1988) 4707-4715. 2579.

${ }^{13}$ M. Zintl and R. McWilliams. "Improved optical tomography device." Review of Scientific Instruments 65(1994) 2574-

${ }^{14}$ D. A. Edrich, R. McWilliams and N. S. Wolf. "Single beam laser induced fluorescence technique for plasma transport measurements." Review of Scientific Instruments 67(1996) 2812-2817.

${ }^{15}$ E. Browne. Table of Isotopes, C. M. Lederer and V. S. Shirley, ed. 7th ed., Wiley, 1978.

${ }^{16}$ C. R. Bingham, M. L. Gaillard, D. J. Pegg, H. K. Carter, R. L. Mlekodaj, J. D. Cole, and P. M. Griffin. "Collinear fast-beam laser spectroscopy experiment: measurement of hyperfine structure and isotope shifts in Xe II." Nuclear Instruments and Methods 202(1982) 147-152.

${ }^{17}$ G. Borghs, P. De Bisschop, R. E. Silerans, M. Van Hove, and J. M. Van den Cruyce. "Hyperfine structures and isotope shifts of the $5 d^{4} D_{7 / 2}-6 p{ }^{4} P_{5 / 2}^{0}$ transition in xenon ions." Zeitschrift fur Physik A 299(1981) 11-13.

${ }^{18}$ H. Kopfermann. Nuclear Moments. Academic Press, 1958.

${ }^{19}$ S. Svanberg. Atomic and Molecular Spectroscopy: Basic Aspects and Practical Applications. 3rd ed., Springer-Verlag, 2001.

${ }^{20}$ L. Bröstrom, A. Kastberg, J. Lidberg, and S. Mannervik. "Hyperfine-structure measurements in Xe II." Physical Review A 53(1996) 109-112.

${ }^{21}$ C. C. Candler. Atomic Spectra and the Vector Model. Van Nostrand, 1964.

${ }^{22}$ P. A. Jansson. Deconvolution of images and spectra. Academic Press, 1997.

${ }^{23}$ T. B. Smith. Deconvolution of ion velocity distributions from laser-induced fluorescence spectra of xenon electrostatic thruster plumes. Ph.D. dissertation, Dept. of Aerospace Engineering, University of Michigan, January 2003.

${ }^{24}$ R. Fletcher and C. M. Reeves. "Function minimization by conjugate gradients." Computer Journal 7(1964) 141-154.

${ }^{25} \mathrm{~J}$. R. Shewchuk. An introduction to the conjugate gradient method without the agonizing pain. School of Computer Science, Carnegie Mellon University, 1994. http://www-2.cs.cmu.edu/ jrs/jrspapers.html.

${ }^{26}$ B. E. Beal. Clustering of Hall effect thrusters for high-power electric propulsion applications. Ph.D. dissertation, Dept. of Aerospace Engineering, University of Michigan, January 2004.

${ }^{27}$ G. J. Williams Jr., T. B. Smith, F. S. Gulczinski and A. D. Gallimore. "Correlating laser-induced fluorescence and molecular beam mass spectrometry ion energy distributions." Journal of Propulsion and Power 18(2002) 489-491. 